\title{
Interactive Language Learning through Speech-Enabled Virtual Scenarios
}

\author{
Hazel Morton, Nancie Gunson, and Mervyn Jack \\ Centre for Communication Interface Research, School of Engineering, University of Edinburgh, Edinburgh EH9 3JL, UK \\ Correspondence should be addressed to Hazel Morton, hazel.morton@ed.ac.uk
}

Received 30 May 2012; Accepted 5 September 2012

Academic Editor: M. Carmen Juan

Copyright () 2012 Hazel Morton et al. This is an open access article distributed under the Creative Commons Attribution License, which permits unrestricted use, distribution, and reproduction in any medium, provided the original work is properly cited.

This paper describes the evaluation of an educational game designed to give learners of foreign languages the opportunity to practice their spoken language skills. Within the speech interactive Computer-Assisted Language Learning (CALL) program, scenarios are presented in which learners interact with virtual characters in the target language using speech recognition technology. Two types of interactive scenarios with virtual characters are presented as part of the game: the one-to-one scenarios which take the form of practice question and answer scenarios where the learner interacts with one virtual character and the interactive scenario which is an immersive contextualised scenario where the learner interacts with two or more virtual characters within the scene to complete a (task-based) communicative goal. The study presented here compares learners' subjective attitudes towards the different scenarios. In addition, the study investigates the performance of the speech recognition component in this game. Forty-eight students of English as a Foreign Language (EFL) took part in the evaluation. Results indicate that learners' subjective ratings for the contextualised interactive scenario are higher than for the one-to-one, practice scenarios. In addition, recognition performance was better for these interactive scenarios.

\section{Introduction}

When learning a foreign language, opportunities for interaction in the target language can be limited. Unlike most other school subjects, language learning requires oral practice. A student studying a language in high school may receive only a few hours of language class per week and may have very limited one-on-one time with the teacher of the class. In the classroom situation, it may not be possible, due to time restrictions and resources, for the teacher to engage in a spoken dialogue with every student. However, it is necessary for language learning that the learner has an interlocutor with whom to interact.

It has been found that classroom exercises which are detached from real-life issues or activities fail to help the learner use the target language $[1,2]$. Learning activities therefore focus less on the (correct) use of forms in a contextfree learning environment in favour of using the language for a communicative purpose. Games are used in language learning to stimulate motivation and to create communicative opportunities for learners [3]. Interactivity and individual action are fundamental properties of games and can be related to communicative approaches to language learning [4], where the focus is on communicating in the target language for meaning.

Simulation games are frequently used in language learning. Simulations offer learners the opportunities to be actively involved in the interactions in the target language. Simulations and role play follow an interactional view of language. The interactional perspective "sees language as a vehicle for the realization of interpersonal relations and for the performance of social transactions between individuals" [5, page 17]. Simulations offer the learner the opportunity for the development of their language in a given social context. Research has suggested that simulations can facilitate second language acquisition; learners acquire language when they are exposed to comprehensible input, and they are actively involved and have positive affect [6].

It has been suggested that language teaching should shift towards experiential learning where the learning occurs in contextualised or situational environments, and language forms are introduced during social activities [7]. Using 
simulations in a learning environment can offer students an opportunity for experiential learning, as they support "learning by doing" approaches [8]. In addition to exposure to comprehensible input, simulations can offer the learner the opportunity of expressing themselves in the target language within a relevant context. The interactions that learners make are part of the process of language learning and can have an effect on their language development. The use of simulations in an educational environment allows the learner to experience situations in which their decisions have real and immediate consequences.

The interaction hypothesis [9] states that conversational interaction between a learner and, for example, a native speaker can facilitate the learner's development as the learner can be involved in negotiated interaction which then gives them comprehensible input in the target language (L2). The approach described here builds on the interaction hypothesis to create situations in which a learner can engage in meaningful spoken interactions with the computer, and whose interactions can be negotiated in order that the learner can develop their oral language in the L2. In this game, learners can engage in negotiated interaction with the virtual agents. The agents act as the "audience" for the learner's oral language output. Learners' output in language learning is thought to be a necessary condition for language learning [10]. One of the key aspects in the design of the game was to consider the application as being one of the conversational participants in the L2 interaction. "It is useful to view multimedia design from the perspective of the input it can provide to learners, the output it allows them to produce, the interactions they are able to engage in, and the L2 tasks it supports" [11]. The virtual agents are able to offer feedback to the learner's utterances as well as continue the dialogue with the learner through a number of conversational turns within the defined context of the language lesson.

The language learning game described here creates contextualised scenarios which are simulations of real world situations in which learners can engage with virtual characters to practice their oral language skills. The game uses speech recognition, so the learner can interact with the system through speech in a simulated, and suitably constrained, environment; virtual agents and virtual worlds are used to depict a context in which the learner can engage in a conversational dialogue with the computer. Within this context, two different kinds of interaction are available between the learner and the virtual characters: the one-to-one scenarios and the interactive scenarios. The two scenario types differ in their pedagogical intent. The one-to-one scenarios allow the learner to interact with one virtual character in a series of question and answer turns, which represent a practice session of the key linguistic topics and forms for the given topic. The interactive scenario allows the learner an opportunity to interact with two or more virtual characters within a highly contextualised scene in order to accomplish a relevant task. In this study, we sought to investigate the one-to-one scenarios separately from the interactive scenario as the different contexts of interaction could influence the way in which the learner chooses to interact with the system. Firstly, there may be differences in user attitudes and response types the learner makes as they progress through each of the scenarios in the "lesson." Secondly, there may be differences across these scores and the recognition performance between the scenario types.

Previous studies have been conducted using the program to investigate overall attitudes towards using the program for learners of Italian and Japanese [12], to investigate motivation [13], and to investigate help strategies [14]. The purpose of the study described in this paper is to investigate learner perceptions of and attitudes towards the speech interactive CALL game and how these attitudes change as the learner progresses through the game and for the different speech interactive scenarios. This paper first describes the various components of the speech interactive game and then provides details on the design. The experimental evaluation is then presented. Data are presented on learner attitudes towards interacting with the characters. In addition, learner response data on the utterances made while interacting with the characters are presented together with performance data on the speech recognition component of the different scenario types within the game.

\section{Speech Interactive Language Learning}

An important aspect of learning a foreign language is becoming comfortable and confident with speaking in the target language. However, for many learners of foreign languages; there are limited opportunities for practicing speaking in the target language. By using speech recognition technology, CALL programs can create more opportunities for learners to practice speaking in the target language and develop their oral language skills. Although a speech-enabled CALL program could not replace one-to-one interaction with a native speaker, it may be possible to offer a more realistic and beneficial simulation in a way that is absent from most CALL materials.

Virtual modelling can create animated characters with which users can interact, and virtual environments can be modelled in which the interaction takes place, which may increase the sense of immersion. Thus, immersive virtual scenarios can be entered by learners as places to practice their oral language skills in the target language.

2.1. Virtual Agents. Virtual agents endowed with speech recognition competency, otherwise known as embodied conversational agents, can introduce a social aspect to the interface. The term "embodied conversational agents" refers to humanlike or cartoon-like animated characters that often appear in computer interfaces [15]. The agents are endowed with conversational capabilities primarily through speech output generation (either synthesised or recorded speech), speech recognition software, and natural language processing. These agents are thought to "anthropomorphise" the interface by bringing lifelike qualities to the interaction: they can react to user's speech input and are capable of verbal and nonverbal output. 
Virtual agents or animated agents are being increasingly used in computer user interfaces to offer a more personalised interaction between human and computer. Animated agents have also been used in pedagogical applications in which such pedagogical agents are defined as "lifelike characters that facilitate the learning process" [16]. Pedagogical agents have been used in a number of applications such as a $2 \mathrm{D}$ animated agent used to support students during medical problem solving activities in a web-based learning environment [17] and a 3D animated agent immersed in a simulated virtual world used as a teaching aid for engineering students [18]. Agents have also been used in a language training program for US soldiers $[16,19]$. In this application, the agents reside in a highly contextualised 3D environment in which the interactions take place.

Early research in the use of animated agents in pedagogical applications has shown such agents to be effective when used in tutoring systems in which they can improve the learning experience by engaging students in effective conversations with their agent [20]. In addition, it has been shown that students who learn with an animated agent work harder to understand the lesson material than students who learn in a text-based environment [21].

The use of animated agents within the contextualised virtual world used in the CALL game described here offers the learner an opportunity for one-to-one conversation, designed to contribute to an enhanced learning experience. Animated pedagogical agents have been shown to "increase the computer's ability to engage and motivate students" [22]. In the context of CALL, it has been suggested that it may be important for learners to have an audience for their linguistic output so that the learners can "attempt to use the language to construct meanings for communication rather than solely for practice" [11]. In this way, animated pedagogical agents could serve as the cyber audience for language learners' output.

2.2. Virtual Worlds. Virtual reality has been defined as "an event or entity that is real in effect but not in fact [23]". In their use of virtual environments, users may experience presence, that is, the subjective sense of "being there" in the virtual world [24]. The underlying assumption is that if users experience such a sense of presence in a virtual environment, they will come to behave in the virtual environment in a way that is similar to the way they would behave in a similar environment in the real world. Indeed, Transfer appropriate processing asserts that memory is optimum in retrieval environments which closely match the environment in which the mental process was encoded [25]. Thus, if learners have the opportunity to practice skills in the virtual environment that are similar to skills needed in the real world task, the skills learned there are likely to carry over to similar situations in the real world. Virtual environments offer features that are superior to video presentations because of the sense of presence in the environment created through the manipulation of certain aspects of that environment and because of the interactivity they allow. The virtual worlds presented to the learner in the game reported here offer a highly contextualised environment in which the learner can first observe the interactions between the virtual agents and then can enter the environment as an active dialogue participant.

2.3. Speech Recognition in CALL. The role of automated speech recognition technology in CALL programs has been explored for more than a decade, having been used for pronunciation practice in CALL programs [26-29] or to help learners with their fluency or conversation skills $[16,30-$ 33]. Many CALL programs which utilise speech recognition technology for language learning are based on strategies where the learner selects their (spoken) response from a finite list offered by the CALL program itself. Such strategies have been used to effect in pronunciation training programs, although their utility is lower in the context of conversational programs. For programs designed to offer learners the opportunity to practice their conversational skills, providing a predefined list of utterances from which the learner can select their spoken response is limiting, as the learner is thereby restricted to use the utterances offered in the list rather than having the opportunity to formulate their own (even incorrect) utterances.

A challenge for speech interactive CALL is to create opportunities for learners to interact through speech with the program in a way that pushes them to develop their language skills by being able to formulate their own responses rather than choose from a preselected list. The CALL game described in this paper permits learners to respond openly, rather than selecting a response from a given list, by engaging in a spoken dialogue with the virtual characters, albeit within a defined lesson context.

\section{Language Learning Game Design}

The aim in the game is for learners to engage in a dialogue with the virtual characters within a defined context. The lesson content design used in this program adheres to a taskbased approach in which the language is used to perform communicative tasks. Each individual lesson has an ultimate communicative goal (e.g., ordering food and drinks in a café), and each lesson focuses on the language required for this communicative goal. In the design of the lessons, the communicative goal of the lesson is defined and the necessary language to complete the goal of the lesson is then scripted into the scenarios.

Based on the interaction hypothesis [9], the virtual characters are designed to offer modifications of their input in cases where the learner appears to be having difficulties. Interaction provides learners with opportunities to receive comprehensible input and feedback [9, 34, 35]. Further, interaction allows learners to make changes to their own linguistic output $[10,36]$. In the game, the learners are not told in advance what to say, nor are they given a finite list from which to choose their utterances; the speech recognition grammars are programmed with predicted responses for each individual stage of the dialogue, accounting for grammatical and some ungrammatical responses. This design 
poses a challenge for the speech recognition component with respect to how accurately the system is able to process the learners' responses.

Implicit feedback is preferable to corrective feedback for speech interactive CALL systems, as implicit feedback is likely to minimise potential problems resulting from imperfect speech recognition [37]. Feedback in the game is given implicitly in the form of recasts and reformulations. If the system detects that the learner has made an error in their utterance, the animated character recasts the learner's utterance. If the learner does not respond, the animated character repeats the question. If the system detects that the learner has given an answer that is not appropriate to the given stage, the system "rejects" this and the animated character reformulates the question, possibly offering a hint to the learner. These feedback strategies allow the dialogue with the learner to continue without explicit reference to a problem. This has the advantage of continuing the flow of the dialogue (and where necessary giving the learner another opportunity to respond, or implicitly correcting their response), and by being implicit in the feedback, this minimises attention to any potential errors made by the speech recognition component.

The program offers the learner three scenario types within each "lesson": observational, one-to-one, and interactive. Supplementary materials are also available to the learners to access if they require vocabulary, grammar files, a transcription of the observational dialogue, and cultural information.

3.1. The Observational Scenario. In order to provide the learner with the concepts required for the communicative goals of the lesson, the game contains an observational scenario which depicts a spoken dialogue between multiple characters within the defined context of the lesson. The key linguistic constructions relevant to the scene and which are useful for the learner in the subsequent scenarios are presented in the observational scenario.

The observational scenario contains a number of virtual characters situated within the virtual world (e.g., in a graphical representation of a "railway station" or "restaurant"). The virtual characters exhibit speech, gesture and facial animation, and manipulation of objects in the environment. They "speak" by means of prerecorded audio files. The virtual characters interact with each other in the target language, utilizing key linguistic structures appropriate for the given context. The learner observes this interaction and has control over the interaction in that they can pause, stop, and restart the dialogue and can access features to assist their understanding if required (e.g., vocabulary information or subtitles of the dialogue). The observational scenario presents the language relevant to the scene in a contextualised environment. The use of this observational scenario also gives the learner the opportunity to become accustomed to the virtual world in which they will become an active participant in the interactive scenarios, and it offers aural practice of the language within the contextualised environment.
3.2. The One-to-One Scenarios. Building on the exposure to the observational scenario, the one-to-one scenarios offer the learner the opportunity to practice key linguistic features by responding to questions on the related topic prior to their participation in the interactive scenario. In this way, they act as training scenarios for the learner before their ultimate immersion in the interactive scenario. The one-to-one scenarios involve one virtual character who asks the learner a series of questions relevant to the lesson topic. There is no movement around the scene, thus allowing the learner's focus to be on the virtual character and the questions asked. These short excerpts of dialogue are designed to ask the learner key questions related to the topic and feature a controlled degree of repetition in the questioning, as well as instructional support, mainly in the form of (implicit) feedback in the learner's responses.

When learners hear and comprehend language, the input is thought to be held briefly in their short-term memory and can be replaced with any forthcoming input unless the learner can focus their attention so that further mental processing can occur [38]. This further mental processing has been described as the process of going from input to intake [39]. Therefore, it is important in instruction to consider how to create opportunities for learners to be exposed to repeated occurrences of new language input, giving learners more opportunities to attend to the input they are exposed to, because the more the student pays attention to the input, the more the student is thought to learn [38]. Therefore, the shorter dialogue excerpts used here are designed to expose the learner to the structures of the language a number of times in order that they have more opportunities to pay attention to these structures.

In this research, participants completed two one-to-one scenarios: About Train Times and Journey Details. In the About Train Times scenario, the virtual character asks the learner some questions about the departure and arrival times of trains in Great Britain. To the side of the character on the screen is a timetable depicting the times. In the Journey Details scenario, the virtual character first asks where the learner would like to go. This dialogue stage is accompanied by a pop-up of a map of Great Britain, with 6 cities in each detailed. Following an appropriate response (i.e., the learner gives the name of a city, either in a one-word form, phrase, or full sentence response), the character then proceeds to ask about the departure and arrival times of the train to that city and the platform from which the train departs (again a timetable is displayed for these dialogue stages to the side of the virtual character). Following the completion of all four stages in the scenario, the character then summarises all the responses.

The one-to-one scenarios incorporate various levels of instructional support for the learner, both through spoken audio prompts from the virtual character and also in the form of text help menus within the scenes for cases where the learner is experiencing some difficulties. Additionally, the virtual tutor character offers implicit spoken feedback to the learner when the learner's utterance has been ungrammatical. As the one-to-one dialogue scenarios offer individualized practice of key linguistic features, they have to cater to 
individual learner preferences and abilities and have to be able to deal with a variety of response types from the learner, such as one-word, phrase, or full sentence responses. In cases where the response was not appropriate for the given dialogue stage, or the learner has not given a response, the system initiates the reformulation strategy, which in this case would first give the learner another opportunity to respond to the same question and subsequently give a hint to the learner if necessary.

For example,

$$
\begin{aligned}
& \text { Virtual Character: Where would you like to go? } \\
& \text { Learner: (no response made) } \\
& \text { Virtual Character: Where would you like to go? } \\
& \text { Learner: Um. } \\
& \text { Virtual Character: I would like to go to Oxford. }
\end{aligned}
$$$$
\text { Where would you like to go? }
$$

In cases where the learner makes a response that is appropriate for the particular dialogue stage, but makes a grammatical error or responds with a one-word reply, the system initiates the recast strategy where the virtual character recasts the learner's response in a full sentence before moving on to the next dialogue stage. This provides additional input to the learner in the form of implicit feedback.

For example,

$$
\begin{aligned}
& \text { Virtual Character: Where would you like to go? } \\
& \text { Learner: I go to Oxford. } \\
& \text { Virtual Character: I see. You would like to go to } \\
& \text { Oxford. }
\end{aligned}
$$

It should be noted that this full sentence recast approach is only implemented in the one-to-one training scenarios. The more immersive interactive scenario did not use a full sentence recast for cases where the learner did not produce a full sentence utterance.

3.3. The Interactive Scenario. The interactive scenario provides the learner with the most immersive and true-tolife simulation of the given environment in which they can practice their spoken language skills. The learner's participation is necessary for the interaction between all dialogue participants (the learner and the virtual characters) in this scene to continue. In the interactive scenario, the most likely flow of possible interactions is scripted, and alternative paths are created, which allows a variety of inputs from the learner. The learner interacts through speech with the agents, but in contrast with the one-to-one scenarios, the agents respond appropriately through a number of conversational turns, which results in an appropriate dialogue relevant to the scene.

The camera viewpoint is from the learner perspective, as if through the learner's eyes, and hence a virtual representation (avatar) of the learner is not depicted. Instead, the viewpoint creates the impression that the learner is in the scene with the characters. The viewpoint also changes as the dialogue moves forward. As an example, the initial

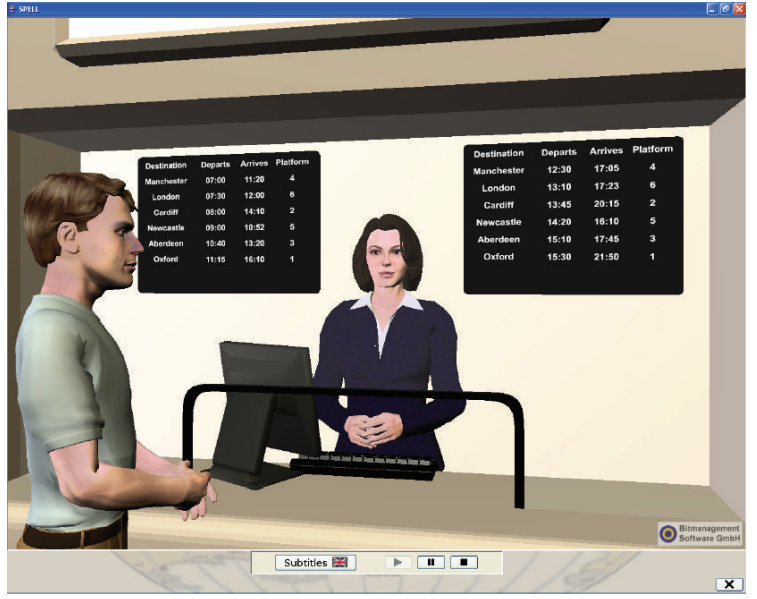

FIGURE 1: Virtual characters in interactive scenario "At the Station."

viewpoint is from the front of the scene, as if walking into the railway station ticket office. The viewpoint then pans into the room towards the ticket agent with a slight upand downward motion to indicate the learner walking to the ticket booth. Multiple interactions occur between the "friend" character and the "ticket seller" character with the learner and with each other. Addressing each other is made with gaze behaviours between the characters and the learner. The distance between the learner and the characters and the angles at which they are standing are such that it is obvious when the learner is being addressed and when the characters are in interaction with each other. This firstperson perspective, which changes dynamically throughout the dialogue, was designed to create a sense of immersion for the learner.

Within this multiagent environment, it is apparent who is being addressed at any one time by the gaze of the virtual characters. The learner is directly addressed by the characters, and the characters are able to hand items within the scene to the implied body of the learner. In order to further stimulate immersion and participation, various items are used in the scene, relevant to the given context. When the learner orders the train tickets, a departure timetable board appears on the screen behind the ticket seller agent. Once the learner successfully orders their required tickets and relevant time of departure, the ticket seller character passes the tickets to the learner. This represents the feedback from a game perspective, whereas the virtual character's reformulations constitute feedback from a linguistic-instructional perspective.

Interacting in the interactive scenario allows learners to practice within the virtual setting the key transactional language necessary for train tickets in the target language. Figure 1 depicts the virtual characters in the interactive scenario.

The goal of the interactive scenario is to purchase tickets to the learner's preferred destination in the host country. The ticket seller asks the learner where they would like to go and subsequently takes them through a series of questions 
in order to sell the train ticket. The first question asked is an open question: Hello, how can I help you? This open question allows a number of responses in the grammar files: destination, number of tickets, and ticket type (single or return). Therefore more advanced learners can try out more complex responses, which contain more than one piece of information.

However, the system is also designed to accept any one of those pieces of information if that is what the learner supplies. If the learner is unable to respond to the open question, the dialogue moves into direct questioning which requires a simple yes or no response: Would you like to buy tickets? From here, the dialogue then directs the learner into a series of questions to determine their requested ticket purchase. The potential destinations from which the learner can choose are constrained to a total of six. These are depicted on the timetable screens above the ticket counter. Each destination has two corresponding departure times. Once the learner selects one of the given destinations, the ticket seller then asks which train they would like to take. The corresponding departure times grammar files are then selected in the code. In the cases where the learner has difficulty in selecting a departure time, a reformulation strategy is used where the ticket agent then offers the learner the choice of these two departure times. For example, if the learner selects "Oxford" as their destination but has not been able to select their preferred departure time, the ticket agent then asks: At what time would you like to leave? At 11:15 or at $3: 30$ ?

Once the learner has completed all questions relevant to the ticket purchase, the ticket seller hands the learner the required number of tickets.

3.4. Agent Animations. The agents are created in Virtual Reality Modelling Language (vrml) format with joints and body parts suitable for conversion to H-Anim 1.1 format, which allows the agents to be fully animated. Agent animations such as nods of acknowledgement and hand gestures were deemed important in creating the appearance of a realistic conversation with the agent. In the creation of a virtual agent, appropriate facial expression and gestures can be added to give a lifelike quality to the agent. These nonverbal behaviours are an important part in the perception of believability of the agent. Gestures can give an added dimension to the agents' speech. With gesture, the agent can indicate objects within their virtual context through deictic gestures, can refer to other agents in the scene or to the user of the application, and can draw users' attention to aspects of the virtual context. Facial animations can offer the user some insight into the agent's state; raised eyebrows can indicate surprise, a smile can indicate happiness, and a frown can indicate confusion. In this way, gestures and facial animation benefit the listener in that the listener can read into these non-verbal communications some information which is not expressed in the agent's speech. This is potentially useful for a language learner, as the learner may be able to interpret the agent's facial animations in instances of communicative difficulty; for example, if the agent frowns when they have not understood what the user has said. The agent may also display some functional gestures within the scene. Additionally, the agent may display some listening animations when the user is speaking. The agents and animations used here were able to display the gestures and expressions required for each scene; however, they were somewhat rudimentary. For future applications, an off-theshelf product is being considered (Complete Characters (http://www.rocketbox-libraries.com/) which should allow a more sophisticated look to our characters.

3.5. Speech Recognition Component. In creating the conversational dialogue, a semantic interpretation approach was adopted. In order to facilitate a spoken dialogue with the learner, the system must understand the semantic interpretation of the learner's utterance. This can be achieved by including task-relevant semantic information in the grammar used by the speech recognition component so that the outcome of the speech recognition process is not only a literal transcription of the utterance, but also an interpretation of its meaning. In this design, the semantic information is expressed within the grammar in the form of slot-value assignments, where the recognition of a particular word or phrase leads to the filling of a semantic slot with the information contained in that word or phrase. In addition, the game is designed to offer feedback to the learners on their responses. For this to occur, it must also be able to identify ungrammatical utterances that have been predicted and preprogrammed into the speech recognition files such that if the system detects that the learner has made an error in their utterance, the virtual character recasts the learner's utterance.

In the game, we used a commercially available speakerindependent recogniser (Nuance v8.0), not developed specifically for L2 learning. Therefore the recogniser's basic components, including its acoustic models and its language model, were not trained on nonnative speaker data. Given the state of the art of ASR applications for L2 learning, this approach might not seem evident. Using a speech recogniser that has been trained on non-native speaker data is seen as preferable in ASR-based CALL applications [40]. Much interest is focused on the area of speech recognition programs using non-native speaker models (e.g., $[41,42])$. Acoustic models for language learning applications have been trained on L2 speech only [43] or L1 (first language) and L2 (target language) models used in parallel [44]. However, since our overall project aim was to create language games in a variety of L2 languages, equally with a variety of different L1 backgrounds, we used a commercially available recogniser that supports recognition engines for many different languages.

As described, the speech recogniser used in this game was trained primarily on native speaker models. In this recognition component, we were unable to change the acoustic models. However, we adapted the recogniser lexicon to include alternative pronunciations for each language pair, in consultation with language teachers. (It should be noted that this approach is somewhat limited in comparison 


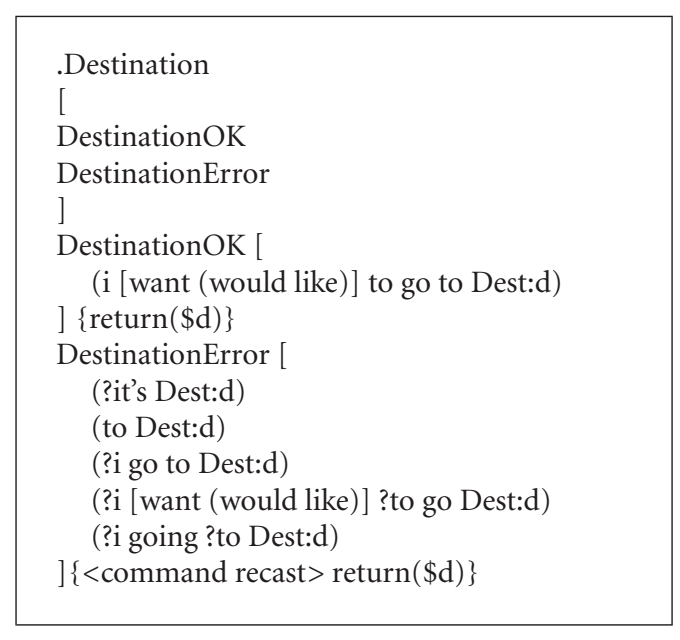

Figure 2: "At the station" recognition grammar extract.

to mixed acoustic models as only those phonemes present in the L2 acoustic models could be used in adaptation of possible L1 transfer errors.)

In addition, the recognition grammars (the language model) in the program were created specifically for nonnative speaking learners using the lessons, including both grammatical and ungrammatical utterances constrained to each stage in the interaction; these recognition grammars were coded by hand by the authors. By using individual recognition grammars for each stage, the possible utterances at each given dialogue stage in a scenario are constrained, thus limiting the list from which the recogniser attempts to make a match. Figure 2 depicts a simplified sample grammar from the railway lesson for the dialogue stage "Where would you like to go?"

This example details the approach taken at every stage in the dialogue between the system (virtual character) and the learner. In this example, the top level grammar ("Destination") calls two sub-grammars: DestinationOK and DestinationError. DestinationOK contains a full sentence, grammatically correct response to the given question (such as "I would like to go to Oxford"). DestinationError contains accepted responses to the question, which may be incomplete sentences (such as "Oxford") or which may contain grammatical errors. Grammatical errors were accounted for in the grammar recognition files to reflect the kinds of errors that might be made by the learners. For example, grammatical errors included preposition omission and subject verb agreement. For example, "I want to go Oxford" is included in the recognition files, and is flagged as containing a preposition omission error. The recast command is triggered if the utterance is within the "error" category.

\section{Evaluation of the Speech Interactive Scenarios}

The experimental evaluation sought to investigate learner attitudes towards the different speech interactive scenarios presented in the game and how these attitudes change as the learner progresses through the game and how they differ between the one-to-one practice scenarios and the immersive interactive scenario. In addition, investigation is made of the types of responses the learners make when interacting with the virtual characters in the scenarios and the accuracy with which the speech recognition component handled the learner responses.

4.1. Participants. A total of 48 students of English as a foreign language took part in the evaluation of the game; all the students recruited from the same junior high school in Beijing, China, and the evaluation took place on location at the school. In this evaluation, there were 22 males and 26 females. Participants were aged between 14 and 15 years at the time of the evaluation and had been studying English in school for an average of 6.8 years. All participants in this study came from the same junior high school in Beijing.

4.2. Experimental Procedure. Participants were first given a short tutorial on using the program (using the navigation and functionality controls, accessing the supplementary materials). Following this, the participants were asked to attempt various aspects of the "At the railway station" lesson. The participants were asked to watch the observational scenario, then try two of the one-to-one scenarios (here referenced as $\mathrm{O}-\mathrm{O} 1$ and $\mathrm{O}-\mathrm{O} 2$ ), and then try the interactive scenario (INT). The participants were informed that they could access other features in the program, for example, subtitles or vocabulary, as they wished. The researcher remained present during the program use. After each scenario, the participants were asked to complete an attitude questionnaire. During the interactions, the system automatically logged all the utterances made by the learners in their interactions for analysis of learners' response type and to investigate the recognition performance in this context.

4.3. User Attitude Questionnaires. User attitude questionnaires were administered after each scenario experienced in by the learners. The usability questionnaire was created in order to gather attitude data to each of the scenarios that the participants experienced. The questionnaires contained items which focused on affective issues, engagement issues, and issues relating to the interaction with respect to the dialogue itself and with respect to the content within the interaction. The attributes are presented in the following.

\section{Affective issues:}

(1) degree of control felt by the learner when talking with the character(s),

(2) degree of embarrassment when talking with the character(s),

(3) extent to which learner felt relaxed when talking with the character(s),

(4) extent to which learner felt stressed when talking with the character(s). 
TABLE 1: Overall mean scores speech enabled scenarios.

\begin{tabular}{|c|c|c|c|}
\hline Questionnaire Statement & $\begin{array}{c}\text { “O-O1" } \\
(\text { Mean = 5.03) }\end{array}$ & $\begin{array}{c}\text { “O-O2” } \\
(\text { Mean = 5.43) }\end{array}$ & $\begin{array}{c}\text { Interactive } \\
(\text { Mean }=5.58)\end{array}$ \\
\hline I felt in control when talking to the character. & 4.50 & 4.90 & 5.08 \\
\hline I felt embarrassed when talking to the character. & 4.31 & 5.25 & 5.52 \\
\hline I felt relaxed talking to the character. & 4.52 & 5.58 & 5.58 \\
\hline I felt stressed talking to the character. & 4.42 & 4.96 & 5.50 \\
\hline I enjoyed interacting with the character. & 5.15 & 5.46 & 5.81 \\
\hline I prefer speaking English in class, rather than interacting with the character. & 4.75 & 5.19 & 5.31 \\
\hline I would be happy to talk to the character again. & 5.54 & 5.77 & 6.19 \\
\hline I felt that this interaction was useful for my learning of English. & 6.33 & 6.33 & 6.31 \\
\hline I felt I always understood what the character said. & 6.06 & 6.23 & 6.38 \\
\hline I felt I always knew how to respond to the character. & 5.31 & 5.65 & 6.06 \\
\hline I felt that the character did not understand what I said. & 4.49 & 5.27 & 5.25 \\
\hline I felt the character was difficult to understand. & 5.58 & 5.84 & 5.90 \\
\hline I felt that the level of the language was difficult for me to understand. & 5.81 & 6.08 & 6.13 \\
\hline I felt that this dialogue was too easy for me. & 3.63 & 3.44 & 3.15 \\
\hline
\end{tabular}

Engagement issues:

(5) extent of enjoyment of interacting with the character(s),

(6) preference for speaking target language in class,

(7) readiness to talk with the character(s) again,

(8) usefulness of interaction for learning language.

Interaction issues (conversational):

(9) extent of understanding what the character(s) said,

(10) extent of knowing how to respond,

(11) extent of being understood.

Interaction issues (content):

(12) extent of difficulty to understand content,

(13) extent of difficulty of language level,

(14) extent of difficulty of dialogue.

The questionnaire consisted of a series of short simple statements, each with a set of tick boxes on a Likert [45] seven-point scale labelled from "strongly agree" through "neutral" to "strongly disagree." The polarity of the statements is balanced to avoid the response acquiescence effect, where respondents may have a natural tendency to agree with proposals. The set of 14 statements was used in the questionnaire for the one-to-one and interactive scenarios. The questionnaire is a self-administered questionnaire and had been translated into Mandarin for students to complete themselves.

When analysing the results, responses to the questionnaire are first given a numerical value from 1 to 7 ; these values are then normalised for the polarity of the statements such that a "strongly agree" response to a positive statement is given a value of 7 , whereas a "strongly agree" response to a negative statement is given a value of 1 . After normalisation of the data, the overall attitude for each participant can be calculated as a mean of all of the scores on the items in the questionnaire. These values can then be used to calculate the overall attitude for all items in the questionnaire across all participants in the study. Additionally, mean scores for individual items in the questionnaire can be obtained for all participants.

\section{Results}

5.1. User Attitude Results. An overall mean score of 5.03 (on a 7-point scale) was obtained for the first one-to-one scenario "about train times," an overall mean score of 5.43 for the second one-to-one scenario "about journey details," and an overall mean score of 5.58 for the interactive scenario. Table 1 details the overall mean scores for the speech enabled scenarios.

It can be seen that attitude scores for the individual items increased across the three consecutive speech-enabled scenarios. Repeated measures analysis was conducted across the attitude data for the three speech-enabled scenarios. Comparing the O-O1 against $\mathrm{O}-\mathrm{O} 2$, it was found that each of the affective issues scored significantly higher in the second scenario than in the first. Participants felt significantly more in control $(P=0.033)$; they felt highly significantly less embarrassed $(P=0.000)$; they felt highly significantly more relaxed $(P=0.000)$; and they felt significantly less stressed $(P=0.011)$. The affective issues are significantly better in the second one-to-one scenario. It appears that as the participants become more accustomed to the interaction there is a positive effect on their affective state.

Additionally, a preference for speaking the language in class, in comparison to speaking with the animated characters, was highly significantly less in the second scenario than in the first $(P=0.000)$, and the feeling that the 
TABLE 2: Response type.

\begin{tabular}{lccccc}
\hline Interaction & Participants & Utterances & Answer only & Sentence & Verbal non answer \\
\hline O-O1 & 48 & 341 & $64.5 \%$ & $29.6 \%$ & $5.9 \%$ \\
O-O2 & 48 & 316 & $64.9 \%$ & $32.6 \%$ & $2.5 \%$ \\
INT & 48 & 452 & $79.8 \%$ & $19.2 \%$ & $0.9 \%$ \\
\hline
\end{tabular}

character did not understand them was highly significantly less in the second scenario than in the first $(P=0.009)$.

Comparing the $\mathrm{O}-\mathrm{O} 2$ against the interactive scenario (the second one-to-one scenario was completed immediately prior to the interactive scenario) found significant differences amongst some individual items in the questionnaire. Participants felt highly significantly less stressed in the interactive scenario than in the second one-to-one scenario $(P=0.002)$; participants were highly significantly more happy to talk to the agents in the interactive scenario again $(P=0.005)$; participants were highly significantly more confident that they knew how to respond in the interactive scenario $(P=$ 0.009 ).

The interactive scenario scored highly across all items in the questionnaire and significantly so in comparison to the one-to-one scenario for some items. Only one affective attribute (feeling stressed) was significantly higher between the interactive and the second one-to-one in comparison to all affective attributes in the earlier comparison. As learners progress through the scenarios, it would seem that their affective state becomes more positive, and therefore feelings of embarrassment and tension and stress subside. Significant results for feeling happy to talk with the characters again and feeling confident in knowing how to respond to the characters suggest an ease for the learners in their interactions with the agents in the immersive interactive scenarios.

5.2. Response Data. Participants' utterances when interacting with the system were recorded and later transcribed by hand (by human raters) for analysis of response type as well as recognition accuracy. The system also logged the recognition results at each stage of the dialogue.

Participants' utterances were categorised into three response types. As the interaction between characters and learner is a series of question and answer pairs, the shortest response type that facilitates the conversation is "answer only." This is often a one word answer (e.g., two) or a phrase response (e.g., two tickets please). The second response type employed is "sentence" which contains a main verb (e.g., I would like to buy two tickets to Oxford). The third response type is "verbal non answer." This final category constitutes responses where the learner has made an utterance (which triggers the recogniser) but does not answer the question. For example, mutterings, thinking aloud, verbal hesitations and nonlexical noises (e.g., coughs) are included in the "verbal non answer" category.

Table 2 details the response types for the participants in the evaluation. The response types are given for the individual dialogues within the lesson.

In each scenario, there was a preference for the learner to respond with an answer only response. However, this
TABLE 3: In-grammar and out-of-grammar user input.

\begin{tabular}{lccc}
\hline Interaction & Utterances & IG & OOG \\
\hline O-O1 & 341 & $47.2 \%$ & $52.8 \%$ \\
O-O2 & 316 & $50.3 \%$ & $49.7 \%$ \\
INT & 452 & $72.1 \%$ & $27.9 \%$ \\
\hline
\end{tabular}

percentage was much higher in the interactive scenario than in the one-to-one scenarios. Almost a third of responses in the one-to-one scenarios were full sentence responses; whereas, in the interactive scenario only fifth of responses were full sentences. The one-to-one scenarios, with their question and answer practice design, is more conducive to full sentence responses where the learner practices their grammatical constructions. In the interactive scenario, where the immersive nature suggests the learner is interacting in order to accomplish a goal, it is less intuitive to respond with a full sentence construction.

5.3. Speech Recognition Analysis. The accuracy of the speech recognition component is analysed by comparing the transcriptions of learner utterances with the output from the recogniser. The utterances are then grouped into in-grammar and out-of-grammar responses. In-grammar responses (IG) have been defined in the grammar recognition files (i.e., the system developer has predicted and programmed the exact word-for-word response). Out-of-grammar responses (OOG) are utterance strings which have not been included in the recognition grammar files.

Table 3 details the in-grammar and out-of-grammar responses for the participants in the evaluation.

For the two one-to-one scenarios, there was almost an even split between in-grammar and out-of-grammar utterances. That is, just over half of all utterances produced by the learners in the one-to-one scenarios were not predicted by the designers and written into the language model. Given that the recognition grammars were designed to incorporate a variety of responses, both grammatical and ungrammatical, constrained to each individual question in the dialogue, it is problematic that so many learner responses were out-ofgrammar. Investigation of the responses made by the learners which were not included in the recognition grammars is made below (see out-of-grammar recognition analysis).

Looking at the interactive scenario compared with the one-to-one scenarios, it was found that far more utterances (72\%) were in-grammar in the interactive scenario than the one-to-one scenarios. This may reflect the higher incidences of shorter (answer only) responses in the interactive scenarios, given the transactional nature of those scenarios. It does show, however, that learners made responses which fit into 
TABLE 4: In-grammar recognition accuracy.

\begin{tabular}{lccccc}
\hline Interaction & $\begin{array}{c}\text { IG } \\
\text { utterances }\end{array}$ & $\begin{array}{c}\text { Word } \\
\text { for } \\
\text { word }\end{array}$ & $\begin{array}{c}\text { Semantic } \\
\text { value }\end{array}$ & Misrec & Reject \\
\hline O-O1 & 161 & $62.1 \%$ & $71.4 \%$ & $8.1 \%$ & $20.5 \%$ \\
O-O2 & 159 & $67.3 \%$ & $79.2 \%$ & $3.1 \%$ & $17.6 \%$ \\
INT & 326 & $68.7 \%$ & $81.0 \%$ & $1.8 \%$ & $17.2 \%$ \\
\hline
\end{tabular}

the predicted language model more readily in the interactive scenario than in the less contextualised one-to-one scenarios.

\section{In-Grammar Utterances}

The speech recognition outputs for the IG utterances were analysed in terms of both word-for-word recognition and semantic value recognition. As the interaction in the dialogues follows a series of question and answer pairs, a semantic value is logged for each of the learner's utterances. As an example, the question from the program "What time does the train to Newcastle leave?" might elicit the answer "it leaves at six o'clock." In this response, the word-forword recognition is the string "it leaves at six o'clock" which is one of the strings in the grammar; whereas, the semantic value is "six o'clock." If the program recognizes this utterance as "it leaves six o'clock", the word-for-word recognition is wrong but the semantic value is correct. Responses are also categorised where the semantic value is wrong, misrecognised [MisRec] or where the program rejects the utterance (in which case the reformulation strategy is employed giving the learner another opportunity to respond). Table 4 details the recognition accuracy for the ingrammar utterances.

Across the three scenarios, a similar pattern of wordfor-word and semantic value accuracy was found. Accurate recognition of the semantic value allows the dialogue to continue effectively between the system and the learner; the system has "understood" the learner's response correctly. For example, in the data, to the question "Where would you like to go?" one participant answered "I want go Oxford." The system recognised this, incorrectly, as "I want to go Oxford." In this case, the system was able to respond to the participant's answer appropriately (by then asking questions relating to the departure time of the train to Oxford). The correct recognition of the semantic value is useful for the facilitation of the dialogue between the characters and the learner; however, it does not always indicate that the learner's errors have been identified.

A rather high rejection rate of IG utterances was found across the three scenarios. This is not ideal; however, given that the users of the system are non-native speakers of the target language (and of the recogniser's acoustic models), it is perhaps unsurprising. Technological limitations can be mitigated against in the design of the interactions. In this program, the effect of a system reject at any stage in the dialogues is that the character repeats or reformulates the initial question and the learner has another opportunity
TABle 5: Out-of-grammar recognition.

\begin{tabular}{lcccc}
\hline Interaction & $\begin{array}{c}\text { OOG } \\
\text { utterances }\end{array}$ & $\begin{array}{c}\text { Correct } \\
\text { reject }\end{array}$ & $\begin{array}{c}\text { Recog } \\
\text { semantic }\end{array}$ & $\begin{array}{c}\text { Misrec } \\
\text { semantic }\end{array}$ \\
\hline O-O1 & 180 & $67.2 \%$ & $16.7 \%$ & $16.1 \%$ \\
O-O2 & 157 & $63.7 \%$ & $26.1 \%$ & $10.2 \%$ \\
INT & 126 & $69.0 \%$ & $26.2 \%$ & $4.8 \%$ \\
\hline
\end{tabular}

to respond to the question. Although this does not hinder the dialogue between the system and the learner, it may contribute to participants' perceptions of the recognition performance of the application.

\section{Out-of-Grammar Utterances}

Investigation was made on the out-of-grammar utterances made by the participants. It is expected that out-of-grammar utterances should be rejected by the system, as the system is not programmed to "listen" for these utterances. However, although these utterances are out of grammar, the system may misrecognise some of these utterances for something within the recognition grammars. Analysis of the out-ofgrammar utterances classifies the results into three categories: correct rejection of the utterance, recognition of the correct semantic value of the utterance or misrecognition of the semantic value of the utterance. For example, an utterance may contain a mid-utterance repetition, which would not be included in the recognition grammars. However, if the system then misrecognises this utterance for an utterance that is in the recognition grammars and the value of the recognition is accurate, then the system will proceed appropriately. Note that as with the semantic value recognition in the IG utterances, these correctly recognised semantic value utterances would not necessarily trigger the system to offer feedback to the learner in the form of recast. Again, they only indicate those utterances where the dialogue proceeds with the response that the participant intended. Table 5 details the category types for the out-of-grammar utterances across the two groups.

The majority of OOG utterances across all three scenarios were correctly rejected by the system. Such utterances include utterances in the participants' first language (thinking aloud), non-lexical responses, or hesitation noises as well as responses that are inappropriate to the question asked. With regards to these utterances, the system handles the responses by reformulating the question and giving the user another chance to respond. That is, even though the system designer has not predicted the response made here by the learner, by correctly rejecting the utterance, the system handles the response appropriately. A sizeable minority of OOG utterances were recognised with the correct semantic value. These utterances often include short disfluencies in the learner's utterance or self-repairs which entailed the utterance was OOG; however, the system recognised the utterance with the intended semantic value. An example of a self-repair in the data which resulted in the recognition 
of the correct semantic value is the response "nine fifty in the aft-in the evening" which was recognised as "nine fifty in the evening." In these cases, the responses are technically out-of-grammar, however, the outcome of the recognition is an accurate response (as far as the user is aware). It is not possible to account for every possible user disfluency in the language models. Although the design of the recognition grammars incorporates user disfluency at the beginning of an utterance (e.g., "eh nine"), other disfluencies are not programmed into the recognition grammars.

Finally, the one-to-one scenarios produced a sizeable minority of utterances which were misrecognised with the wrong semantic value. The Interactive scenario only produced a very small percentage of such utterances. These are the most problematic as they are likely to cause confusion on the learner's part. Analysis of these OOG misrecognitions highlighted that there were some problems (which also occurred in the IG misrecognition results) with similar sounding time responses. For example, in the data there were multiple misrecognitions of "thirteen" for "thirty," "fourteen" for "forty" and "fifteen" for "fifty," and vice versa. Similar sounding responses can be avoided in the design of the dialogues by careful dialogue planning. However, in cases where it was felt that restricting the dialogue to exclude similar sounding expressions (which in the case of time expressions may not be appropriate), the CALL program designer could mitigate against such potential recognition errors by employing an additional confirmation stage where the virtual character seeks confirmation from the learner on their response (e.g., "Did you say "ten o'clock"?"). Such a strategy may mimic what would occur in a real-life situation if the coparticipant in the dialogue was unsure of what was said.

\section{Discussion}

The results of the evaluation indicate that speech interactive CALL systems are potentially very useful for language learners, despite misrecognitions by the speech recognition component. Analysis of the speech recognition component found in-grammar semantic value accuracy rates of up to $81 \%$ for the interactive scenario. However, word-for-word recognition performance was as low as $62 \%$ indicating that the recognition component is not robust enough to accurately determine exactly what the learner has said (within this open dialogue design). Regardless of recogniser inaccuracies, user attitude results indicate a high level of engagement and enjoyment with using the system. This is in accordance with research into the use of automatic speech recognition in CALL applications [32], which found that despite the limitations of the speech recogniser and the misrecognitions it generated, end users enjoy the interactions with the system and would prefer a speech interactive component to be included in the CALL application. This was also found in a previous evaluation of the software described here [12].

There are limitations to the open dialogue approach used in this program in the more difficult one-to-one scenarios. Contextualised help is provided in the immersive interactive scenario, which lends itself to more natural, shorter responses within the given context which is optimal for the speech recognition technology. The one-to-one scenarios are similar to traditional question and answer response type which are akin to the teacher in class asking a question that they know the answer to already. The interactive scenario is a more genuine question and answer where the responses lead to a communicative goal.

The design of the game, with open speech dialogue is optimal for the immersive contextualised interactive scenario. Users responded naturally in short answers which felt "right" and which was an easier challenge for the speech recognition technology. The interactive scenario design is a good fit for the technology and for the ways in which users interacted with the virtual characters.

Investigation of user responses in this study found that there was a tendency for shorter responses in the interactive scenarios in comparison with the one-to-one scenarios. With regards to coverage in the recognition grammars, participants in this study made responses which fit into the language model of the interactive scenario far more than in the one-to-one scenarios. In designs where the learner has to select a response from a finite list, the recognition grammar is fully constrained to those given responses. However, when there is no list from which the learner can choose their response, the recognition grammars must include a variety of possible responses, both grammatical and ungrammatical. Given the number of out-of-grammar responses was around the same as the in-grammar responses, it would appear that this approach is not usable. However, in the investigation of the out-of-grammar recognition results, we found that the majority of the responses were correctly rejected by the system (thus allowing another chance for the learner to respond), and a sizable minority of responses were recognised with the user's intended meaning.

On the whole, grammar coverage for the interactive scenario was measurably higher than in the one-to-one scenarios. That is, participants in this study produced responses which were included in the recognition grammars much more frequently in the interactive scenario than in the one-to-one scenarios. Therefore, it was easier to predict learner responses in the interactive scenario. In addition, participants tended to produce fewer hesitancies in their responses in the interactive scenario. Observation suggests this may be due to the fact that the physical context and visual cues in the interactive scenario served to make the meaning of the questions clearer, resulting in a higher proportion of in-grammar responses. Moreover, the situational context encourages the use of brief or one-word answers reducing the risk of an out-of-grammar response. For example, in response to the question "How many tickets would you like?" the reply "Two please" would be a natural response in conversation. To respond with a complete sentence such as "I would like two tickets please" in this case is more likely to appear unnatural.

It should be noted that each participant in the experiment experienced these scenarios in the same order. It was important to see if there was any effect on participants' attitudes towards interacting within the scenarios over time 
and more experience. As the Interactive scenario builds upon concepts and constructions which have been practised in the one-to-one scenarios, it might be expected that attitudes towards this scenario are more favourable than towards the one-to-one scenarios.

Allowing the learner to formulate their own responses to the animated agents' questions, rather than selecting from a predefined list, provides a facilitative environment for language learning. However, this poses a serious challenge for both the designer in terms of predicting learners' responses and the recogniser in having to match the response against a potentially lengthy recognition grammar.

The interactive scenario produced higher user attitude scores and better recognition, suggesting that this type of scenario is better suited to the technology limitations. It seems that interactive scenarios, where the communicative activity is highly contextualised, are more conducive to this type of open response dialogue design. While maximising the potential of ASR in speech interactive CALL, such interactive scenarios, or participatory dramas, also serve to motivate learners and reduce their inhibition [46].

The user response types show that learners were producing shorter responses in the interactive scenario and therefore potentially not pushing their linguistic capabilities in the target language in this context. The study shows an overall favourable attitude towards each of the scenarios and in particular the interactive scenario. The benefits to the one-toone scenarios are the extended practice of the key linguistic forms and vocabulary for the given context. However, in future studies, an alternative approach restricting the variety of responses expected may be preferable without negatively impacting the learners' experience.

In this study, the focus was the learners' attitudes towards the scenarios, the responses they made, and the recognition performance. We did not investigate any effect of using the program on the learners' ability in the target language. Learners responded strongly that they felt the interactions were useful for their learning of English (6.3 on a 7-point scale), but no objective data was gathered in this area. Future experiments could include some pre- and post-testing of the key linguistic topics included within the lesson to ascertain any positive effects on learning.

\section{Conclusion: Directions for Future Research with Scenario-Based CALL}

Digital games for learning have the potential to offer an enhanced learning experience if designers consider the reasons why computer games are so engaging for learners. In a review of the literature, Mitchell and Savill-Smith [47] identified a number of reasons why computer games are so compelling for users. First, games are visually seductive in that they "use technology to represent reality or embody fantasy" [48]. They have an ultimate goal and motivate the learner via fun [49] and instant visual feedback [50]. Games provide a complete interactive playing environment [51]. Finally, the ambience information in the digital game creates an immersive experience, sustaining the user's interest in the game [51].

Thus, engaging computer games offer an immersive fantasy or realistic world along with clearly defined achievable goals and instant and observable outcomes. Immediate and interactive feedback is a compelling quality of digital games [52]. A common element in digital games is where the game can "respond seamlessly to a player's input" and therefore offers "real-time game play that shifts and reacts dynamically to player decisions."

Designers of CALL games should look ahead at how commercial games could inspire the design of educational applications in order to make them more engaging and compelling for learners. Adaptation of the interaction or program could be tailored to suit the individual learner's needs. The game or activity must be seen as relevant and appropriate to the individual user. Activities which are designed to be fun and engaging can be found to be tedious and uninteresting by some users [53]. Commercial games are demographically targeted to specific users and styles, whereas resources may restrict such variety in learning games. Customisation of the game could allow the user some control to make changes to, for example, the skill level, speed of interaction, complexity of interaction, or time to respond, in order to make them suitable to the learner's own needs and wants.

Good computer games are "pleasingly frustrating" [50] which are mastered as they are played. Ideally, the game should be played without the need for consulting instructions external to the game. In immersive interactive environments especially, any halting of the game to deploy or exhibit instructions to the user could be detrimental to the immersive aspects of the game. If the system is nonintuitive, or if instructions are necessary for the game to proceed, the user has to quit their presence in the game in order to exit out to receive the instructions.

Taking the video games dimension a step further, learners could inhabit the virtual world as a "surrogate avatar," the virtual character the player is playing [50]. In this way, the learner must take on the mental states they believe the virtual character has in their decisions to achieve the goals of the game. Thus, the learner has the opportunity to experience "alternative identities" [54]. This type of virtual simulation can open up the space to fantasy and thus allow for a more compelling environment for future spoken CALL systems.

\section{References}

[1] A. Firth and J. Wagner, "On discourse, communication, and (some) fundamental concepts in SLA research," The Modern Language Journal, vol. 81, no. 3, pp. 285-300, 1997.

[2] L. van Lier, "From input to affordance: social-interactive learning from an ecological perspective," in Sociocultural Theory and Second Language Learning, J. P. Lantolf, Ed., pp. 245-259, Oxford University Press, Oxford, UK, 2000.

[3] M. Warschauer and D. Healey, "Computers and language learning: an overview," Language Teaching, vol. 31, pp. 57-71, 1998. 
[4] B. H. Sørensen and B. Meyer, "Serious games in language learning and teaching-a theoretical perspective," in Proceedings of the 3rd International Conference of the Digital Games Research Association, pp. 559-566, Tokyo, Japan, 2007.

[5] J. C. Richards and T. S. Rogers, Approaches and Methods in Language Teaching, Cambridge University Press, Cambridge, UK, 1986.

[6] R. Scarcella and D. Crookall, "Simulation/gaming and language acquisition," in Simulation, Gaming and Language Learning, D. Crookall and R. L. Oxford, Eds., pp. 223-230, Newbury House, New York, NY, USA, 1990.

[7] L. van Lier, "An ecological-semiotic perspective on language and linguistics," in Language Acquisition and Language Socialization: Ecological Perspectives, C. Kramsch, Ed., pp. 140-164, Continuum, London, UK, 2002.

[8] K. Facer, "Computer games and learning," NESTA Futurlab Discussion Paper, pp. 1-11, 2005, http://www.nestafuturelab .org/research/discuss/02discuss01.htm.

[9] M. H. Long, "The role of the linguistic environment in second language acquisition," in Handbook of Second Language Acquisition, W. C. Ritchie and T. K. Bhatia, Eds., pp. 413-468, Academic Press, New York, NY, USA, 1996.

[10] M. Swain, "Three functions of output in second language learning," in Principle and Practice in Applied Linguistics: Studies in Honour of H. G. Widdowson, G. Cook and B. Seidlhofer, Eds., pp. 125-144, Oxford University Press, Oxford, UK, 1995.

[11] C. A. Chapelle, "Multimedia CALL: lessons to be learned from research on instructed SLA," Language Learning \& Technology, vol. 2, no. 1, pp. 22-34, 1998.

[12] H. Morton, N. Davidson, and M. A. Jack, "Evaluation of a speech interactive CALL system," in Handbook of Research on Computer-Enhanced Language Acquisition and Learning, F. Zhang and B. Barber, Eds., 2008.

[13] H. Morton and M. Jack, "Speech interactive computer-assisted language learning: a cross-cultural evaluation," Computer Assisted Language Learning, vol. 23, no. 4, pp. 295-319, 2010.

[14] H. Morton, N. Gunson, and M. Jack, "Attitudes to subtitle duration and the effect on user responses in speech interactive foreign language learning," Journal of Multimedia, vol. 6, no. 5, pp. 436-446, 2011.

[15] J. Cassell, "Nudge, nudge, wink, wink: elements of faceto-face conversation for embodied conversational agents," in Embodied Conversational Agents, J. Cassell, J. Sullivan, S. Prevost, and E. Churchill, Eds., pp. 1-27, MIT Press, Cambridge, Mass, USA, 2000.

[16] W. L. Johnson, S. Choi, S. Marsella, N. Mote, S. Narayanan, and H. Vilhjálmsson, "Tactical language training system: supporting the rapid acquisition of foreign language and cultural skills," in Proceedings of the InSTIL/ICALL Symposium on Computer Assisted Language Learning, pp. 21-24, Venice, Italy, 2004.

[17] W. L. Johnson and E. Shaw, "Using agents to overcome difficulties in web-based courseware," in Workshop on Intelligent Educational Systems on the World Wide Web (AI-ED '97), pp. 1-8, Kobe, Japan, 1997.

[18] W. L. Johnson, J. W. Rickel, and J. C. Lester, "Animated pedagogical agents: face-to-face interaction in interactive learning environments," International Journal of Artificial Intelligence in Education, vol. 11, pp. 47-78, 2000.

[19] W. L. Johnson and A. Valente, "Tactical language and culture training systems: Using artificial intelligence to teach foreign languages and cultures," in Proceedings of the 23rd AAAI Conference on Artificial Intelligence and the 20th Innovative
Applications of Artificial Intelligence Conference (AAAI'08/IAAI '08), pp. 1632-1639, Chicago, Ill, USA, July 2008.

[20] J. C. Lester, S. A. Converse, S. E. Kahler, S. T. Barlow, B. A. Stone, and R. S. Bhogal, "Animated pedagogical agents and problem-solving effectiveness: a large-scale empirical evaluation," in Proceedings of the 8th World Conference on Artificial Intelligence in Education, pp. 23-30, 1997.

[21] R. Moreno, R. E. Mayer, and J. C. Lester, "Life-like pedagogical agents in constructivist multimedia environments: Cognitive consequences of their interaction," in Proceedings of World Conference on Educational Multimedia, Hypermedia and Telecommunications, pp. 741-746, AACE Press, Charlottesville, VA, USA, 2000.

[22] W. L. Johnson, J. W. Rickel, and J. C. Lester, "Animated pedagogical agents: face-to-face interaction in interactive learning environments," International Journal of Artificial Intelligence in Education, vol. 11, pp. 47-78, 2000.

[23] M. Heim, The Metaphysics of Virtual Reality, Oxford University Press, Oxford, UK, 1994.

[24] M. Slater, M. Usoh, and A. Steed, "Depth of presence in virtual environments," Presence, Teleoperators and Virtual Environments, vol. 3, pp. 130-144, 1994.

[25] C. D. Morris, J. D. Bransford, and J. J. Franks, "Levels of processing versus transfer appropriate processing," Journal of Verbal Learning and Verbal Behavior, vol. 16, no. 5, pp. 519533, 1977.

[26] W. Menzel, D. Herron, R. Morton, D. Pezzotta, P. Bonaventura, and P. Howarth, "Interactive pronunciation training," ReCALL, vol. 13, no. 1, pp. 67-78, 2001.

[27] J. Dalby and D. Kewley-Port, "Explicit pronunciation training using automatic speech recognition technology," CALICO Journal, vol. 16, no. 3, pp. 425-445, 1999.

[28] M. Eskenazi, "Using a computer in foreign language pronunciation training: what advantages?" CALICO Journal, vol. 16, no. 3, pp. 447-469, 1999.

[29] J. Murray, "Lessons learned from the Athena language learning project," in Intelligent Language Tutors: Theory Shaping Technology, V. Holland M, J. D. Kaplan, and M. R. Sams, Eds., pp. 243-256, Lawrence Erlbaum Associates, Mahwah, NJ, USA, 1995.

[30] J. Bernstein, A. Najmi, and F. Ehsani, "Subarashii: encounters in Japanese spoken language education," CALICO Journal, vol. 16, no. 3, pp. 361-384, 1999.

[31] W. G. Harless, M. A. Zier, and R. C. Duncan, "Virtual dialogues with native speakers: the evaluation of an interactive multimedia method," CALICO Journal, vol. 16, no. 3, pp. 313337, 1999.

[32] M. Holland, J. D. Kaplan, and M. Sabol, "Preliminary tests of language learning in a speech-interactive graphics microworld," CALICO Journal, vol. 16, no. 3, pp. 339-359, 1999.

[33] H. Strik, F. Cornillie, J. Colpært, J. van Doremalen, and C. Cucchiarini, "Developing a CALL system for practicing oral proficiency: how to design for speech technology, pedagogy and learners," in Proceedings of the SlaTE-2009 Workshop, Warwickshire, UK, 2009.

[34] T. Pica, "Research on negotiation: what does it reveal about second-language learning conditions, processes, and outcomes?" Language Learning, vol. 44, pp. 493-527, 1994.

[35] S. Gass, Input, Interaction, and the Second Language Learner, Lawrence Erlbaum Associates, Mahwah, NJ, USA, 1997.

[36] M. Swain, "Communicative competence: some roles of comprehensible input and comprehensible output in its development," in Input in Second Language Acquisition, S. Gass and 
C. Madden, Eds., pp. 235-253, Newbury House Press, Rowley, Mass, USA, 1985.

[37] K. Wachowicz and B. Scott, "Software that listens: it's not a question of whether, it's a question of how," CALICO Journal, vol. 16, no. 3, pp. 253-276, 1999.

[38] C. Doughty and J. Williams, "Pedagogical choices in focus on form," in Focus on Form in Classroom Second Language Acquisition, C. Doughty and J. Williams, Eds., pp. 197-261, Cambridge University Press, Cambridge, UK, 1998.

[39] S. M. Gass, "Integrating research areas: a framework for second language studies," Applied Linguistics, vol. 9, no. 2, pp. 198-217, 1988.

[40] H. Strik, A. Neri, and C. Cucchiarini, "Speech technology for language tutoring," in Proceedings of the Language and Speech Technology Conference (LangTech '08), pp. 73-76, Rome, Italy, 2008.

[41] J. Morgan and S. LaRocca, "Making a speech recognizer tolerate non-native speech through Gaussian mixture merging," in Proceedings of InSTIL/ICALL Symposium on Computer Assisted Language Learning, pp. 213-216, Venice, Italy, 2004.

[42] N. Cylwik, A. Wagner, and G. Demenko, "The EURONOUNCE corpus of non-native polish for ASR-based pronunciation tutoring system," in Proceedings of the SLATE Workshop on Speech and Language Technology in Education, Warwickshire, UK, 2009.

[43] L. Neumeyer, H. Franco, M. Weintraub, and P. Price, "Automatic text-independent pronunciation scoring of foreign language student speech," in Proceedings of the International Conference on Spoken Language Processing (ICSLP '96), pp. 1457-1460, October 1996.

[44] G. Kawai and K. Hirose, "A method for measuring the intelligibility and non-nativeness of phone quality in foreign language pronunciation training," in Proceedings of the International Conference on Spoken Language Processing (ICSLP '98), pp. 1823-1826, Sydney, Australia, 1998.

[45] R. Likert, A Technique for the Measurement of Attitudes, Columbia University Press, New York, NY, USA, 1932.

[46] P. Hubbard, "Interactive participatory dramas for language learning," Simulation and Gaming, vol. 33, no. 2, pp. 210-216, 2002.

[47] A. Mitchell and C. Savill-Smith, "The use of computer and video games for learning. A review of the literature, Ultralab," 2004, http:/gmedia.glos.ac.uk/docs/books/ computergames4learning.pdf.

[48] Becta, "Computer games in education," Project Report, 2001.

[49] C. Bisson and J. Luckner, "Fun in learning: the pedagogical role of fun in adventure education," Journal of Experimental Education, vol. 19, no. 2, pp. 108-112, 1996.

[50] J. P. Gee, "Pleasure, learning, video, games and life: the projective stance," E-Learning and Digital Media, vol. 2, no. 3, pp. 21-223, 2005.

[51] M. Prensky, Digital Game-Based Learning, McGraw Hill, New York, NY, USA, 2001.

[52] K. Salen and E. Zimmerman, Rules of Play: Game Design Fundamentals, MIT Press, Cambridge, Mass, USA, 2004.

[53] S. Franciosi, "A comparison of computer game and language learning task design using flow theory," CALL-EJ, vol. 12, no. 1, pp. 1-25, 2011.

[54] R. Sandford and B. Williamson, Games and Learning: A Handbook From Futurlab, 2005, http://www2.futurelab.org.uk/ resources/documents/handbooks/games_and_learning2.pdf. 

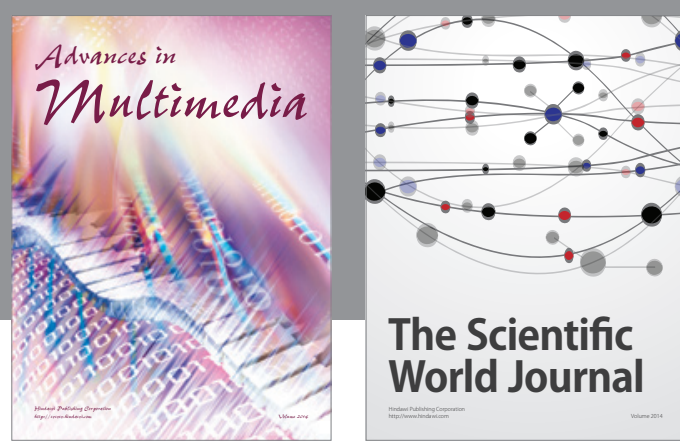

The Scientific World Journal
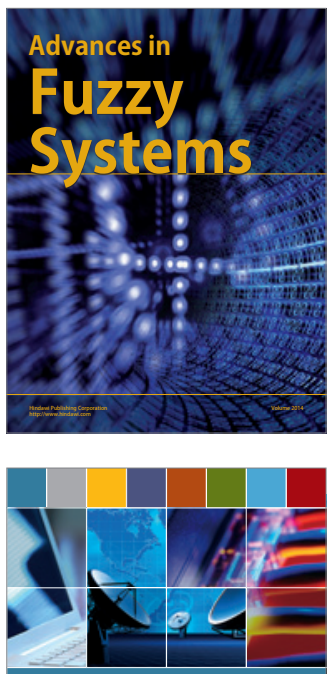

Computer Networks and Communications
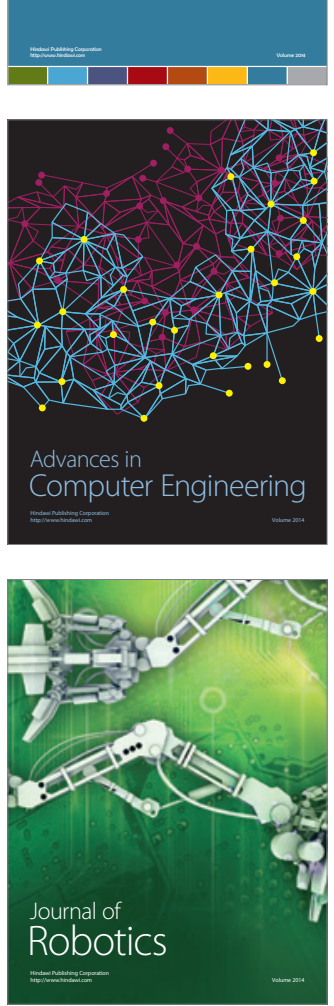
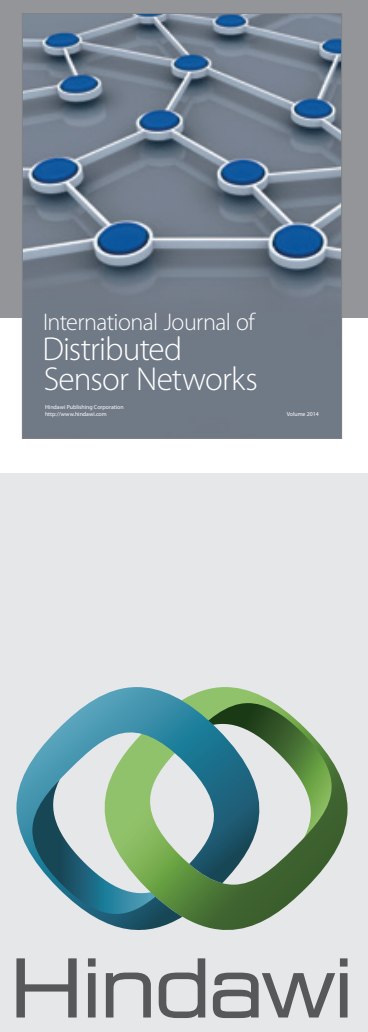

Submit your manuscripts at

http://www.hindawi.com
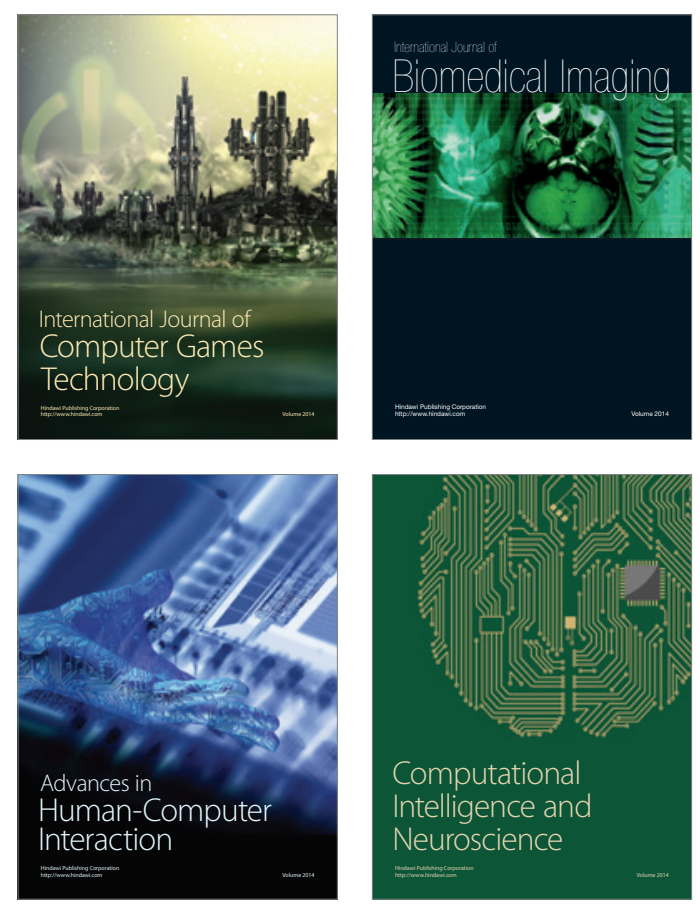
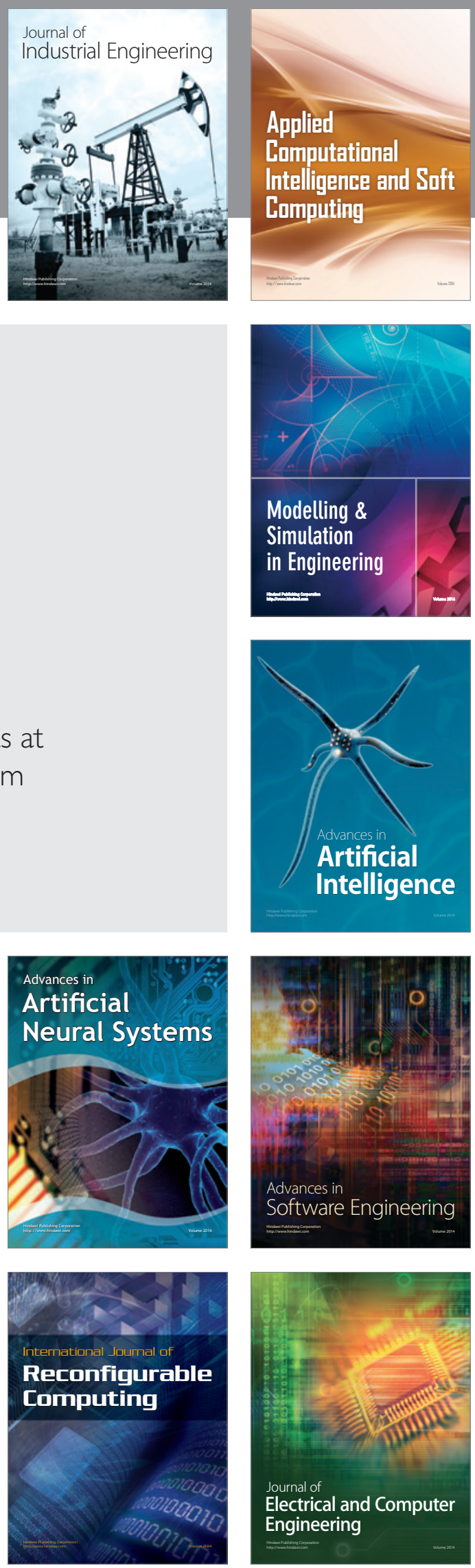